\title{
Ruptured and unruptured aneurysms of the accessory anterior cerebral artery combined with a blood blister-like aneurysm of the anterior communicating artery
}

\author{
Dae-H ee Seo, W on-Cheol Lee, Il-Seung Choe, Sung-Choon Park, Young-Soo Ha \\ Department of Neurosurgery, Myongï Hospital, Kwandong University School of Medicine, Koyang, Korea
}

\author{
Address for correspondence: \\ Dr. Dae-Hee Seo, \\ Department of Neurosurgery, \\ Myongji Hospital, Kwandong \\ University School of M edicine, \\ Koyang, Korea. \\ E-mail-nsdrseo@ kwandong.ac.kr
}

DOI: $10.4103 / 0028-3886.48806$

\begin{abstract}
Abstradt
Anterior communicating artery (Acom) complex and anterior cerebral artery (ACA) are the frequent sites of arterial anomalies. Aneurysms associated with triplicate ACA are rare. Blood blister-like aneurysm (BBA) of the Acom is also very rare. This report presents these two rare types of aneurysms in a 63-year old man who presented with subarchnoid hemorrhage secondary to accessory ACA aneurysmal rupture. During surgery, a BBA of the Acom was also found. Both the aneurysms were treated simultaneously. The BBA bled intraprocedurally but was repaired successfully by neck reconstruction and clipping with the preservation of the parent and the branching arteries. The ruptured accessory ACA aneurysm was clipped together with the artery which had multiple aneurysms distally. For the treatment of a complex aneurysm like this, accurate understanding of the anatomic variations, proper imaging study and extremely careful technique are essential.
\end{abstract}

Key words: Accessory anterior cerebral artery, anterior communicating artery, blood blister-like aneurysm

\section{Introduction}

The accessory anterior cerebral artery (ACA) has been termed as the medial ACA, the median callosal artery (MACC), the superior callosal artery, and the third A2 artery. ${ }^{[1]}$ But the accurate definition of the terms is still lacking. The incidence of triplicate ACA in patients with anterior communicating artery (Acom) aneurysms varies between 4.5-14.2\%. ${ }^{[2]}$ The aneurysmal formation of the triplicate artery is quite rare. We describe a case of ruptured accessory ACA aneurysm at the origin of the artery and multiple aneurysms in clusters along the axis of the artery itself associated with a rare blood blister-like aneurysm (BBA) of the Acom.

\section{Case Report}

A 63-year-old male was admitted with stuporous mental status and was paraparetic on neurological examination. Past medical history was unremarkable. Computed tomography (CT) scan showed thick and diffuse subarachnoid hemorrhage (SAH) [Figure 1a]. The 3D-CTA disclosed an anomalous vessel originating from the Acom complex and passing into the interhemispheric fissure between the two A2 segments. Contralateral A1 seemed hypoplastic. The aneurysms were in a cluster form along the axis of the anomalous artery [Figure 1b].

The morphology of the aneurysms rendered them unsuitable for endovascular treatment and a left 
fronto- temporal craniotomy was performed to occlude the ruptured aneurysms proximally between the A2 on the day of admission. The dissection and exposure were difficult because of severe brain swelling. Paine's point was punctured and approximately $30 \mathrm{cc}$ of cerebrospinal fluid was aspirated to relieve brain swelling. Gyrus rectus was removed subpially and the lamina terminalis was opened. As more blood clots were removed, a dilated, thin-walled ruptured aneurysm at the origin of the accessory ACA and a purplish BBA were seen on the horizontal portion of the Acom. The BBA appeared clippable. The two aneurysms were unidentified and not suspected preoperatively. Postoperative retrospective review of the 3D-CTA image showed a suspicious dimpling on the Acom corresponding to the location of the BBA, but the bulging at the origin of the accessory ACA seemed incompatible with the operative finding [Figure 2]. After clipping with a Yasargil right-angled clip, the BBA was believed to be clipped completely at that time. By a few strokes of suction tip during further
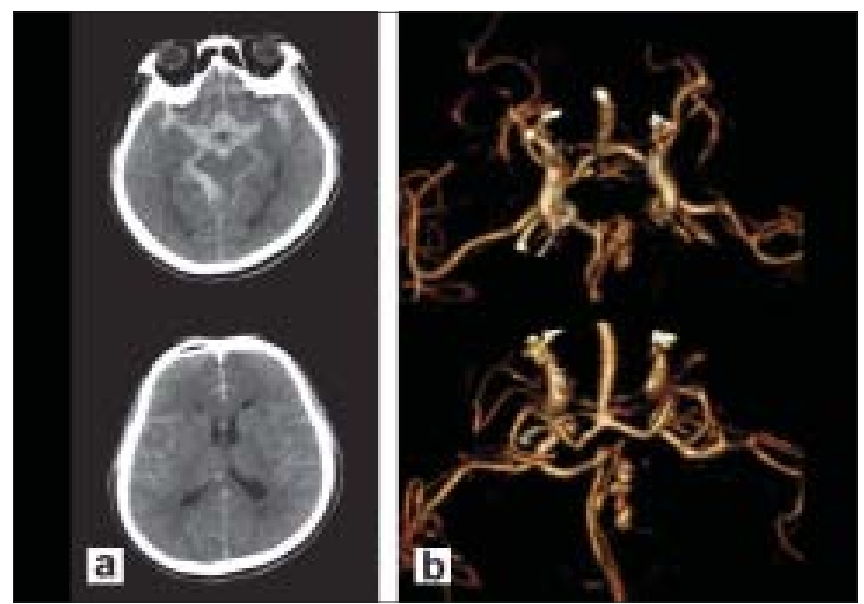

Figure 1: Preoperative noncontrast CT scans (a) obtained on admission showing diffuse SAH and 3D-CTA imaging (b) demonstrating triplicate ACA with abnormal vessel configuration

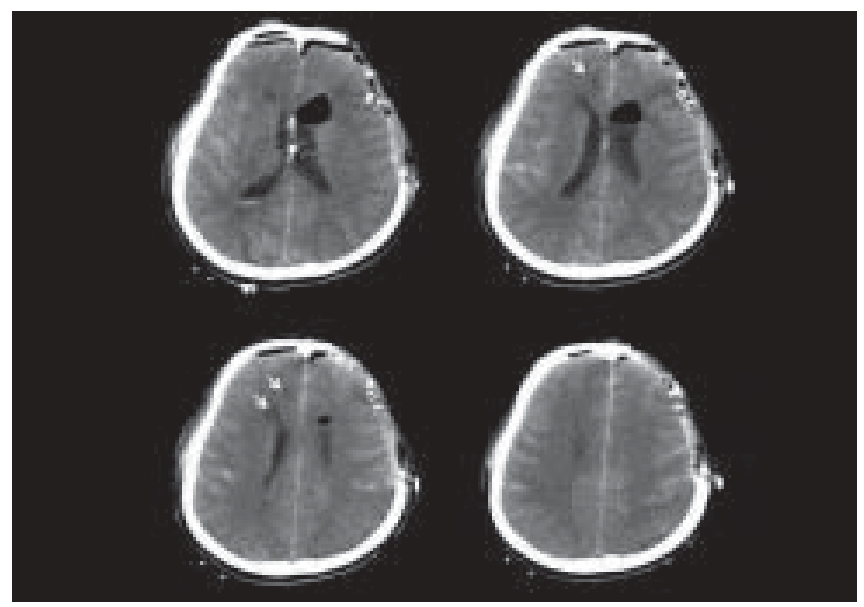

Figure 3: Postoperative noncontrast CT scans showing multifocal, small low-attenuating areas in right frontal lobe (arrows) hematoma removal and dissection, bleeding from the aneurysm was seen. Temporary arterial occlusion on the ipsilateral A1 was used and the aneurysm was coagulated cautiously to reconstruct a neck and eventually reclipped using a miniclip. Not to induce the stenosis of the Acom, the origin of the accessory ACA including the dilated, thin portion of the ruptured aneurysm was clipped precisely by another miniclip. Under ipsilateral A2 artery, the accessory ACA was seen. The further tracking and visualization of the aneurysm and the branch was complicated due to the swelling and we were concerned about a poor result that more retraction might cause. A residual bulging around the Acom-A2 junction [Figure 2 short arrow] and the clipped BBA were reinforced using small pieces of cotton and fibrin glue. The bone flap was not replaced at

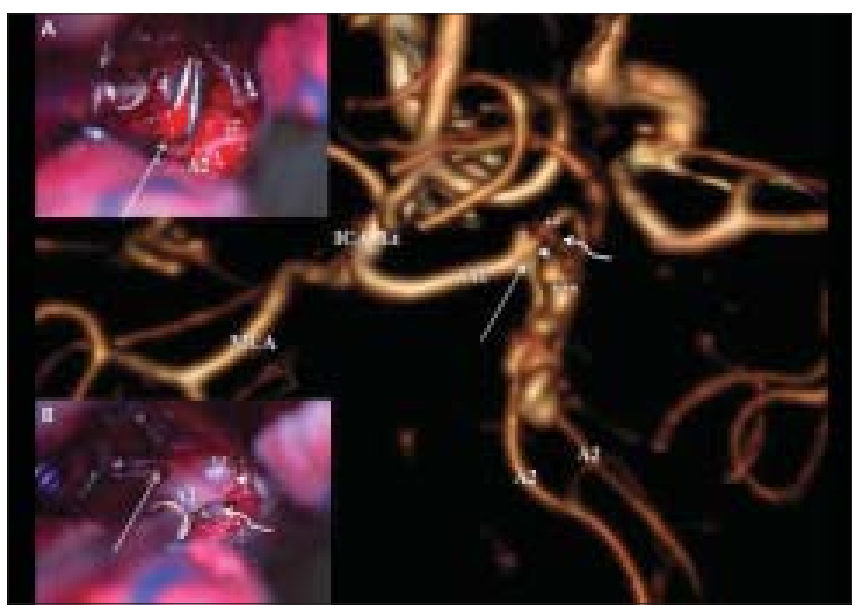

Figure 2: Preoperative 3D-CTA image and the corresponding intraoperative photography (inlets $a$ and $b$ ). (a) The BBA was clipped using a right-angled clip. The accessory ACA origin was dilated and we found that it was the ruptured aneurysm. (b) BBA reclipped after avulsion using miniclip, the accessory ACA and aneurysm was clipped by another clip. *: accessory ACA origin, ${ }^{* *}$ : Acom, ${ }^{* *}$ : aneurysm of the accessory ACA, short arrow: residual, swollen portion after obliteration of accessory ACA origin, long arrow: dimpling of A1-A2 junction, presumed position of BBA, curved arrow: accessory ACA

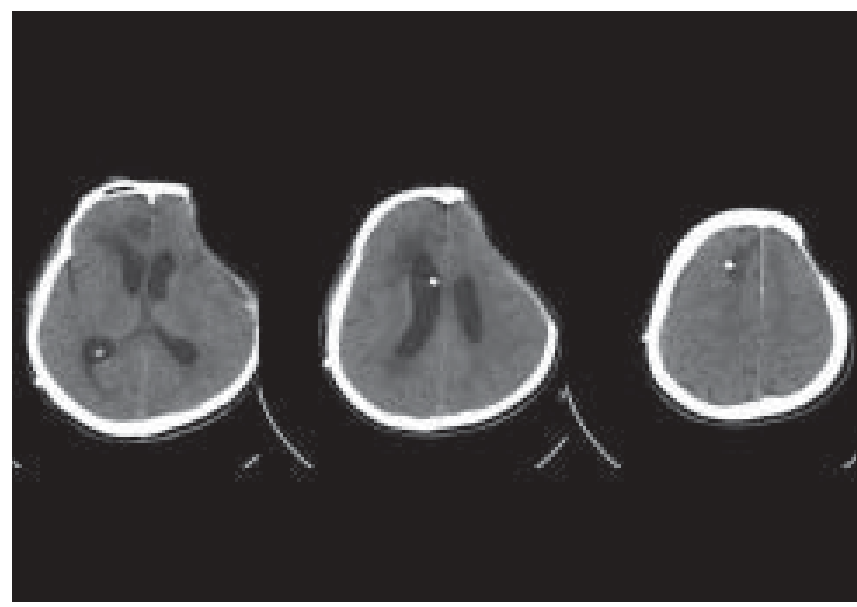

Figure 4: CT scans obtained after a ventriculoperitoneal shunt operation showing persistent multiple infarctions 
the termination of operation in anticipation of cerebral swelling. A few low-density lesions were seen on the postoperative CT scans [Figure 3].

The postoperative course was complicated by vasospasm and systemic complications (pneumonia and deep vein thrombosis), and the patient slowly recovered thereafter. The patient underwent ventriculoperitoneal shunt placement due to the secondary hydrocephalus and was moderately disabled by the frontal lobe dysfunction such as the lack of volition and lower extremity weakness [Figure 4].

\section{Discussion}

The BBA of Acom is a very rare. The BBA is an elusive pathologic entity which was mainly reported in the non-branching site of the internal carotid artery (ICA) and the optimal treatment option remains unclear. ${ }^{[3-5]}$ Recently, five cases of BBA of Acom were reported. ${ }^{[6]}$ Of the 719 nontraumatic SAH treated by the authors over 5 years period, 181 were in the Acom territory, of these five were BBA. During the same period, there were 13 cases of ICA blister aneurysm.

Vascular anomalies of the circle of Willis frequently associated with the aneurysm and the numerous anomalies of the Acom complex and ACA have been described. ${ }^{[2,7]}$ The triplicate ACA is a well documented but not so rare entity (3.3-15\%) $)^{[1,2]}$ Its classification and terminology are not yet clear. The reported incidence of this anomaly in patients with Acom aneurysms varied between 5 and $14.2 \% .^{[2]}$ The aneurysmal formation of the triplicate artery is quite rare and only a few cases have been reported previously. ${ }^{[1,8]}$ In contrast, the incidence of the azygos ACA is more rare than the triplicate ACA, but aneurysmal formation is more common. ${ }^{[1,8]}$

In normal embryonic development, a small embryonic branch MACC arises from the Acom and extends towards the lamina terminalis but it normally involutes by seven weeks gestation as the ACA segment. We cannot definitely say that this aneurysm is MACC or accessory ACA by the definition of Baptista. ${ }^{[7]}$ In the present case, the diameter of the artery was larger than or the same as that of the contralateral A2, the anomalous artery was possibly an accessory ACA. ${ }^{[1]}$ But these terms have been used interchangeably and further tracking of the aneurysm and discerning the presence of the cortical branch was nearly impossible due to swelling and the limitation of the pterional approach itself. As a few infarctions developed postoperatively in the right frontal lobe, one could presume that the artery might be an accessory ACA. Previous investigators have stressed on identifying such accessory vessels to avoid inadvertent clipping of such a vessel, which can result in a devastating stroke. Two cases of poor result caused by inadvertent occlusion of MACC were reported. ${ }^{[9]}$

Despite the possibility of the infarction in the subcortical and cortical territories supplied by this artery, we unavoidably occluded the accessory ACA because of the aneurysms in cluster along its axis. We speculated that there is no other way of preserving this artery. During the operation, trapping of Acom for BBA and accessory ACA aneurysms was considered as a possible therapeutic strategy. But hypoplastic contralateral A1 precluded it. We also considered the interhemispheric approach, but from a point of view that it might be risky to meet the presumably ruptured, malformed aneurysm early in the dissection without securing the proximal artery, we eventually chose the pterional approach. The selection of approach remains a matter of discussion.

\section{References}

1. Morioka M, Fujioka S, Itoyama Y, Ushio Y. Ruptured distal accessory anterior cerebral artery aneurysm: Case report. Neurosurgery 1997;40:399-402.

2. Kwak R, Niizuma H, Hatanaka M, Suzuki J. Anterior communicating artery aneurysms with associated anomalies. J Neurosurg 1980;52:162-4

3. Auguste KI, Ware ML, Lawton MT. Nonsaccular aneurysms of the azygos anterior cerebral artery. Neurosurg Focus 2004;17:E12.

4. Meling TR, Sortenberg A, Bakke SJ, Slettebo H, Hernesniemi J, Sortenberg W. Blood blister-like aneurysms of the internal carotid artery trunk causing subarachnoid haemorrhage: Treatment and outcome. J Neurosurg 2008;108:662-71.

5. Ogawa A, Suzuki M, Ogasawara K. Aneurysms at nonbranching sites in the supraclinoid portion of the internal carotid artery: Internal carotid artery trunk aneurysms. Neurosurgery 2000;47:578-86.

6. Andaluz N, Zuccarello M. Blister-like aneurysms of the anterior communicating artery: A retrospective review of diagnosis and treatment in the five patients. Neurosurgery 2008;62:807-11.

7. Baptista AG. Studies on the arteries of the brain: II-The anterior cerebral artery: Some anatomic features and their clinical implications. Neurology 1963;13:825-35.

8. Kutsuna M, Monden S, Watanabe K. Two cases of distal anterior cerebral artery aneurysm associated with accessory anterior cerebral artery. No Shinkei Geka 2006;34:193-200.

9. Gibbons K, Hopkins LN, Heros RC. Occlusion of an "accessory" distal anterior cerebral artery during treatment of anterior communicating artery aneurysms: Report of two cases. J Neurosurg 1991;74:133-5.

Accepted on 26-01-2009

Source of Support: Nil, Conflict of Interest: None declared. 